\title{
Comparison of Two Novel Swept-Source Optical Coherence Tomography Devices to A Partial Coherence Interferometry-Based Biometer
}

\section{Tommy Chan}

Hong Kong Sanatorium and Hospital

Marco Yu

Singapore Eye Research Institute

Vivian Chiu

Chinese University of Hong Kong

Gilda Lai

Chinese University of Hong Kong

Christopher Leung

Chinese University of Hong Kong

Poemen Chan ( $\nabla$ poemen@gmail.com )

Chinese University of Hong Kong

\section{Research Article}

Keywords: anterior segment optical coherence tomography, partial coherence interferometry, repeatability, agreement, keratometry

Posted Date: December 31st, 2020

DOI: https://doi.org/10.21203/rs.3.rs-132028/v1

License: (c) (i) This work is licensed under a Creative Commons Attribution 4.0 International License. Read Full License

Version of Record: A version of this preprint was published at Scientific Reports on July 21 st, 2021. See the published version at https://doi.org/10.1038/s41598-021-93999-8. 


\section{Abstract}

Purpose To evaluate the repeatability and agreement of cornea and biometry measurements obtained with two swept-source optical coherence tomography (SSOCT) and a partial coherence interferometrybased device.

METHODS This is a cross-sectional study. Forty-eight eyes of 48 patients had three consecutive measurements for ANTERION (Heidelberg Engineering, Germany), CASIAIl (Tomey, Japan) and IOLMaster500 (Carl Zeiss Meditec, USA) on the same visit. Mean keratometry (Km), central cornea thickness (CCT), anterior chamber depth (ACD) and axial length (AL) were recorded. Corneal astigmatic measurements were converted into vector components - J0 and J45. Intra-device repeatability and agreements of measurements amongst the devices were evaluated using repeatability coefficients (RCs) and Bland-Altman plots, respectively.

RESULTS All devices demonstrated comparable repeatability for $\mathrm{Km}(\mathrm{p} \geq 0.138)$. ANTERION had the lowest RC for $\mathrm{JO}$ amongst the devices ( $\mathrm{p} \leq 0.039)$. Systematic difference was found for the $\mathrm{Km}$ and $\mathrm{JO}$ obtained with IOLMaster500 compared to either SSOCTs $(p \leq 0.010)$. The ACD and AL measured by IOLMaster500 showed a higher RC compared with either SSOCTs $(p<0.002)$. Systematic difference was found in CCT and ACD between the two SSOCTs $(p<0.001)$, and in AL between ANTERION and IOLMaster500 ( $p<0.001)$, with a mean difference of $1.6 \mu \mathrm{m}, 0.022 \mathrm{~mm}$ and $0.021 \mathrm{~mm}$, respectively.

CONCLUSIONS Both SSOCTs demonstrated smaller test-retest variability for measuring ACD and AL compared with IOLMaster500. There were significant disagreement in keratometry and AL measurements between the SSOCTs and PCl-based device; their measurements should not be considered as interchangeable but the disagreement could be refined by readjustment of intraocular lens constant in clinical practice.

\section{Introduction}

The advancement of surgical techniques and intraocular lens (IOL) design has greatly improved the outcomes of cataract surgery but also raised patients' expectations for refractive-error free vision. Accurate measurements of axial length (AL), cornea power, astigmatic axis and other anterior segment parameters are crucial for IOL power calculation ${ }^{1}$, especially with the growing popularity of toric IOL implantation to correct preexisting corneal astigmatism at the time of cataract surgery. The IOLMaster (Carl Zeiss Meditec, Jena, Germany) was the first commercially available optical biometry device and has been used widely for IOL power calculation ${ }^{2,3}$. The IOLMaster500 is based on the principle of partial coherence interferometry $(\mathrm{PCl})$ and have demonstrated accurate measurement for IOL power calculation in both routine and complicated cataract cases ${ }^{4,5}$.

Anterior segment ocular coherence tomography (OCT) has the advantage of high tissue penetration, high scanning speed and has the ability to identify both anterior and posterior surfaces with high repeatability ${ }^{6}$. OCT is emerging as a new modality for corneal tomographic analysis ${ }^{7}$. The development 
of swept-source OCT allows quick, one-station, detailed imaging of the cornea, the anterior chamber, as well as the anterior and posterior surfaces of the lens with improved image resolution and scan speed ${ }^{8,9}$. Since the first generation CASIA SS-1000 (Tomey, Nagoya, Japan), the technology continues to improve. For instance, compared with CASIA SS-1000, the new CASIAll offers a faster scan-speed (50,000 vs. 30,000 A-scans/s) and a higher transverse resolution (800 A-scans/B-scan vs. 256 A-scans/B-scan) for $360^{\circ}$ imaging of the ACA using 18 evenly-spaced radial scans over 36 angle locations. The CASIAll (introduced in 2016-2017) and the ANTERION (introduced in 2019, Heidelberg Engineering, Heidelberg, Germany) are two swept-source OCTs that were developed recently.

The purpose of this study was to compare the repeatability and agreement between ANTERION, CASIAII and IOLMaster500 for measurements of keratometry and anterior chamber depth (ACD). Central corneal thickness (CTT) measurements obtained from ANTERION and CASIAll were compared. Axial length measurements obtained from IOLMaster500 and ANTERION were also compared.

\section{Methods}

This was a cross-sectional study conducted at The Chinese University of Hong Kong, Department of Ophthalmology and Visual Science between June to September 2019. Eyes that had previous ocular surgery (including cornea refractive surgery and lens extraction) were excluded. Apart from cataract, eyes with ocular disease including corneal pathologies (e.g. cornea ectasia, pterygium) infectious disease (e.g. infective keratitis, viral conjunctivitis), and /or problem with dry eyes were excluded. Patients with myopia of more than - 6.0 D and visual acuity of worse than Snellen 6/12 were also excluded. Written informed consents were obtained from all subjects. The study was conducted in accordance with the ethical standards stated in the 2013 Declaration of Helsinki and approved by Hong Kong Kowloon Central Research Ethics Committee with written informed consent obtained.

\section{IOLMaster500}

The IOLMaster500 (Carl Zeiss Meditec, Jena, Germany) utilizes the principle of PCI to measure the AL. It evaluates the keratometry with a six-point telecentric technique and an image-based slit lamp system for ACD measurements. It does not provide lens thickness nor CCT measurement. It measures AL from the anterior corneal surface to the retinal pigmented epithelium, along the line of sight.

\section{CASIAII swept-source OCT}

The CASIAll swept-source OCT (Tomey, Nagoya, Japan) is a form of Fourier-domain OCT that utilizes a swept-source wavelength of $1310 \mathrm{~nm}$ as the light source and a photodetector to detect wavelengthresolved interference signal ${ }^{9}$, with improved in image resolution, scan speed, width, and depth ${ }^{8,9}$. At a scan speed of 50,000 A scan/s and an axial resolution of $<10 \mu \mathrm{m}$, it allows multiple high-resolution, up to 256 cross-sectional images of the entire anterior segment and angle to be captured within 3 seconds. The maximum scan width is $16 \mathrm{~mm}$ with a scan depth of $13 \mathrm{~mm}^{10}$. It also provides placido corneal topography and automatic measurement software. With the measuring mode of corneal map and lens 
biometry, 16 radial cross-sectional images, with 800 A-scan per line sampling, a scan width of 16 mm and a scan depth of $11 \mathrm{~mm}$ can be delivered with a scan speed of 0.3 second. It only images the anterior segment with no AL measurement.

\section{ANTERION swept-source OCT}

The ANTERION swept-source OCT (Heidelberg Engineering, Heidelberg, Germany) is another form of Fourier-domain OCT that offers a fast scan-speed of 50,000 A-sans/second. It utilizes a $1300 \mathrm{~nm}$ light source to offer an axial resolution of $<10 \mu \mathrm{m}$. Compared with the CASIAll, it provides a wider scan width (up to $16.5 \mathrm{~mm}$ wide) and a deeper scan depth range $(14 \pm 0.5 \mathrm{~mm})^{11}$. This allows visualization of detail corneal, anterior chamber, angle and lens (both anterior and posterior surfaces). The four different in-built imaging Apps - Cornea App, Cataract App, Metrics App and Imaging App - allows a comprehensive examination of the anterior segment imaging, corneal topography and tomography, anterior segment biometry, IOL calculation and AL measurement in a single scan. With the ANTERION Cornea App, 65 radial B-scan images (256 A-scans per B-Scan) are acquired in less than 1 second and the data can generate corneal maps of $8 \mathrm{~mm}$ in diameter ${ }^{12}$. It measures the distance between the anterior corneal surface and the retinal pigment epithelium, along the line of sight, as the $\mathrm{AL}^{12}$.

\section{Imaging and measurements}

One randomly selected eye of each subject was imaged by all three instruments. Each eye was scanned 3 times for each instrument to obtain clear images of the anterior and posterior corneal surfaces. The sequence of measurement recording between the ANTERION, CASIAII and IOLMaster500 was not fixed. The time elapsed between measurement devices included a short break for the patient to relax for tear film recovery and to avoid fatigue. All measurements were performed by a single experienced technician (G.L.) and were taken under dim room illumination. Patients were asked to blink in between consecutive scans to produce an optically smooth tear film, thereby improving the reflectivity of the cornea. During the imaging, the subjects were asked to fixate at an internal fixation target. To avoid lid artifact, the technician would retract the upper and lower lids of the participant while taking the imaging. To ensure stable corneal conditions, patients were asked to withhold soft contact lens wear for 2 weeks before the evaluation; none of our patients worn hard contact lens.

Power vector analysis was conducted for obtaining vectors along the $0^{\circ}$ and $45^{\circ}$ meridians according to the following equation: $\mathrm{JO}=(-[\mathrm{Ksteep}-\mathrm{Kflat}] / 2 \cos 2 \mathrm{a})$, and $\mathrm{J} 45=(-[\mathrm{Ksteep}-\mathrm{K} f \mathrm{lat}] / 2 \sin 2 \mathrm{a})$, for comparison in a Cartesian coordinate system. ${ }^{13}$ Ksteep, Kflat, and a represent the steep keratometry, flat keratometry, and axes values, respectively. J0 represents astigmatic component along the vertical meridian (with-the-rule or against-the-rule astigmatism), while J45 represents oblique astigmatism.

R 3.2.5 (R Foundation, Vienna, Austria) was used for statistical analysis. Repeatability coefficients (RCs) was used to evaluate the repeatability of measurements obtained by the IOLMaster500, CASIAII and ANTERION. RC is defined as the $95 \%$ confidence limit of the difference of measurement between examinations, which is equal to 
$1.96 \sqrt{(2 \times \text { pooled test }- \text { retest variance })}$.

A high RC value represents a low test-retest variability, and vice versa ${ }^{14}$.

Comparison of RCs for parameters between the CASIAll vs ANTERION, ANTERION vs IOLMaster500 and CASIAll vs IOLMaster500 were evaluated by empirical bootstrap resampling with 2000 replicates. The first attempted measure of each subject is evaluated. Bland-Altman plots were used to assess the agreement between measurements of the two devices. Differences between the measurement values were plotted against the mean values of the measurements, and the $95 \%$ limits of agreement (LoA), which is equal to the mean difference $\pm 1.96 \times S D$, were evaluated. Systematic differences of each parameter between the two devices were compared using the t-test. Proportional bias was investigated by linear regression of the difference in values measured by the two devices. A $P$ value less than 0.05 was considered statistically significant. False discovery rate, which measures the percentage of false discovery due to random error, was evaluated for multiple statistical tests with the threshold of P-value $<0.05^{15}$.

\section{Results}

A total of 48 eyes of 48 patients were included. The mean age was $57.6 \pm 14.8$ years. Table 1 summarizes the repeatability outcomes of the parameters obtained from ANTERION, CASIAII and IOLMaster500. These include the mean keratometry $(\mathrm{Km}), \mathrm{J} 0$ and $\mathrm{J} 45$ vector components of astigmatism, ACD (measured from epithelium to anterior lens surface), CCT (for the two OCT devices only), and AL (for ANTERION and IOLMaster500 only). Both ANTERION and CASIAll demonstrated comparable test-retest reproducibility for measurements of $\mathrm{Km}$ and CCT ( $p \geq 0.138)$. The J0 vector component of astigmatism measured by CASIAll showed a significantly greater RC $(p=0.002)$ compared with the J0 measured by the ANTERION. The repeatability of the J45 vector component of astigmatism were similar across all the devices $(p \geq 0.072)$. RCs were greater for the measurements obtained from IOLMaster500 compare with those obtained by either OCTs although significant differences were found only in ACD and AL measurements $(p \leq 0.002)$. 
Table 1

Repeatability outcomes for biometric measurements obtained using the ANTERION, CASIA II and IOLMaster500

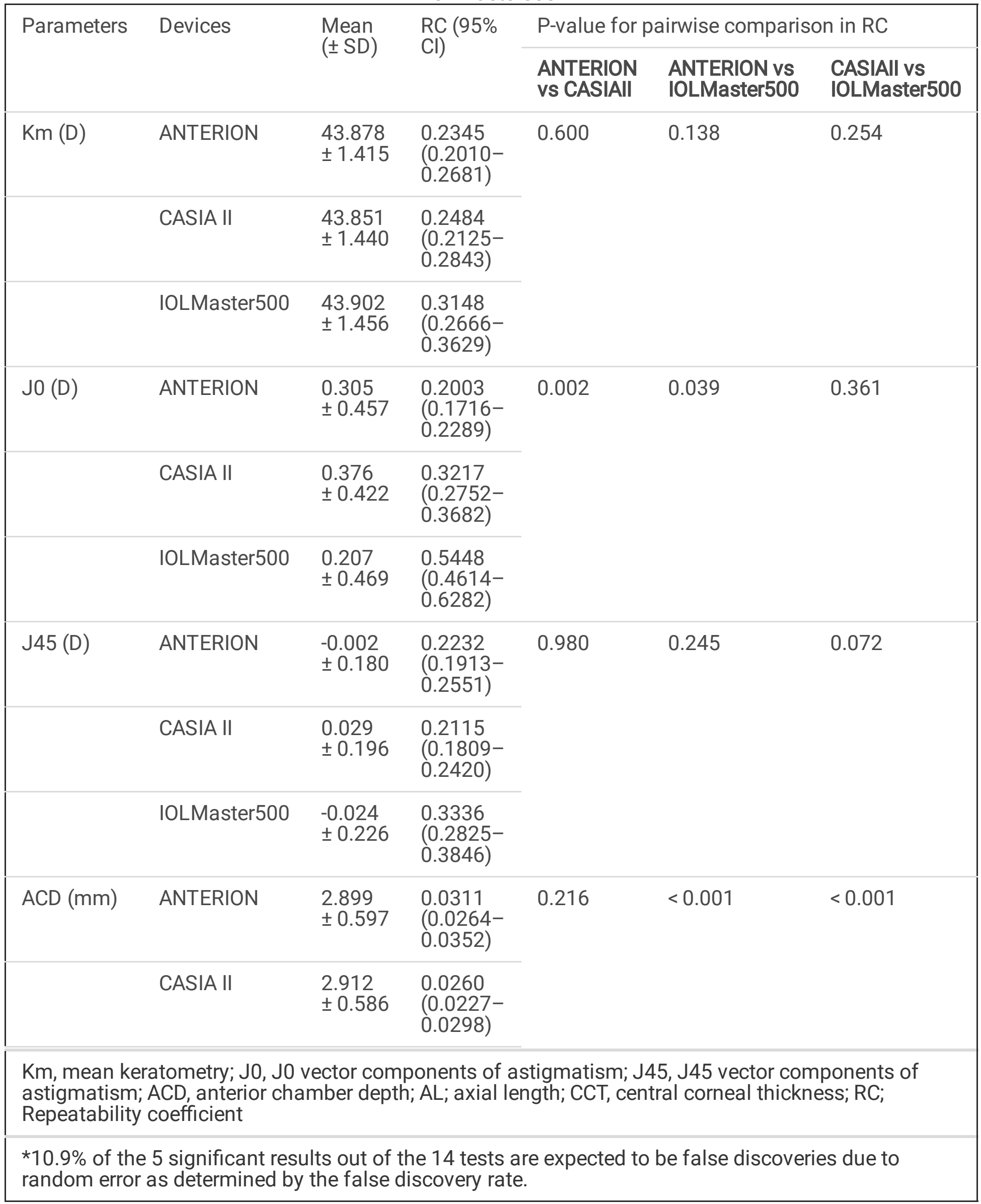




\begin{tabular}{|c|c|c|c|c|c|c|}
\hline & IOLMaster500 & $\begin{array}{l}2.932 \\
\pm 0.563\end{array}$ & $\begin{array}{l}0.1947 \\
(0.1652- \\
0.2241)\end{array}$ & & & \\
\hline \multirow[t]{2}{*}{ ССТ $(\mu \mathrm{m})$} & ANTERION & $\begin{array}{l}543.17 \\
\pm 32.20\end{array}$ & $\begin{array}{l}2.1759 \\
(1.8648- \\
2.4869)\end{array}$ & \multirow[t]{2}{*}{0.575} & \multirow[t]{2}{*}{ / } & \multirow[t]{2}{*}{ / } \\
\hline & CASIA II & $\begin{array}{l}543.02 \\
\pm 33.45\end{array}$ & $\begin{array}{l}2.3223 \\
(1.9868- \\
2.6579)\end{array}$ & & & \\
\hline \multirow[t]{2}{*}{$\mathrm{AL}(\mathrm{mm})$} & ANTERION & $\begin{array}{l}23.683 \\
\pm 1.137\end{array}$ & $\begin{array}{l}0.0154 \\
(0.0130- \\
0.0178)\end{array}$ & \multirow[t]{2}{*}{ / } & \multirow[t]{2}{*}{0.002} & \multirow[t]{2}{*}{ / } \\
\hline & IOLMaster500 & $\begin{array}{l}23.881 \\
\pm 1.323\end{array}$ & $\begin{array}{l}0.0260 \\
(0.0221- \\
0.0299)\end{array}$ & & & \\
\hline \multicolumn{7}{|c|}{$\begin{array}{l}\text { Km, mean keratometry; J0, J0 vector components of astigmatism; J45, J45 vector components of } \\
\text { astigmatism; ACD, anterior chamber depth; AL; axial length; CCT, central corneal thickness; RC; } \\
\text { Repeatability coefficient }\end{array}$} \\
\hline
\end{tabular}

Table 2 shows the systematic differences and the 95\% LoA between ANTERION versus CASIAll, ANTERION versus IOLMaster500, and CASIAll versus IOLMaster500 measurements. Systematic differences were found in CCT and ACD between ANTERION and CASIAll $(p<0.001)$. However, only the ACD measurement showed a proportional bias $(p=0.018)$ and not the CCT measurement $(p=0.149)$.

There were systematic differences for the $\mathrm{Km}$ and the $\mathrm{J} 0$ vector component between either OCT devices and the IOLMaster500. The mean difference of $\mathrm{Km}$ between ANTERION and IOLMaster500 was $-0.115 \mathrm{D}$ $(p=0.002$ ) (span of $95 \%$ LoA was $0.945 \mathrm{D}$ [range: -0.588 to $0.357 \mathrm{D}$ ]) and the mean differences of J0 was $0.153 \mathrm{D}(\mathrm{p}<0.001)$ (span of $95 \%$ of LoA was $0.997 \mathrm{D}$ [range: -0.346 to $0.651 \mathrm{D}$ ]). There was no proportional bias between the two devices ( $p \geq 0.104)$. The mean differences of $\mathrm{Km}, \mathrm{J} 0$ and $\mathrm{J} 45$ vectors of astigmatism between CASIAll and IOLMaster500 were - $0.093 \mathrm{D}(\mathrm{p}=0.010)$ (span of 95\% of LoA was $0.908 \mathrm{D}$ [range: -0.532 to $0.376 \mathrm{D}$ ]), $0.195 \mathrm{D}(\mathrm{p}<0.001)$ (span of $95 \%$ of LoA was $1.158 \mathrm{D}$ [range: -0.384 to $0.774 \mathrm{D}]$ ), and $0.067 \mathrm{D}(\mathrm{p}=0.035)$ (span of $95 \%$ of LoA was $0.830 \mathrm{D}$ [-0.348 to $0.482 \mathrm{D}]$ ), respectively. None of these comparisons showed any proportional bias between the two devices $(p \geq 0.185)$. As for the $\mathrm{AL}$, which was only measured by ANTERION and IOLMaster500, there was a significant mean difference of $-0.021 \mathrm{~mm}(p<0.001)$ (span of $95 \%$ of LoA was $0.068 \mathrm{~mm}$ [range: -0.055 to $0.013 \mathrm{~mm}$ ]) between the two devices; a proportional bias was observed $(p<0.001)$. The Bland-Altman plots of the parameters measured by the three devices were shown in Fig. 1 to 6 . 
Table 2

Systematic differences and proportional biases between different devices

CASIA II vs ANTERION

Systematic Difference

Proportional Bias

\begin{tabular}{|c|c|c|c|c|c|c|}
\hline Parameters & $\begin{array}{l}\text { Mean } \\
\text { differences }\end{array}$ & $95 \%$ LoA & $P$ & $\begin{array}{l}\text { Scaling } \\
\text { difference }\end{array}$ & $\mathrm{R}^{2}$ & $P$ \\
\hline $\mathrm{Km}$ (D) & 0.026 & $\begin{array}{l}-0.349 \text { to } \\
0.401\end{array}$ & 0.351 & 0.001 & $\begin{array}{l}7.33 \times 10^{-} \\
5\end{array}$ & 0.954 \\
\hline J0 (D) & 0.045 & $\begin{array}{l}-0.364 \text { to } \\
0.455\end{array}$ & 0.140 & -0.041 & 0.008 & 0.557 \\
\hline J45 (D) & 0.016 & $\begin{array}{l}-0.256 \text { to } \\
0.289\end{array}$ & 0.419 & 0.058 & 0.007 & 0.581 \\
\hline ACD (mm) & 0.022 & $\begin{array}{l}-0.032 \text { to } \\
0.077\end{array}$ & $<.001$ & -0.016 & 0.116 & 0.018 \\
\hline CCT $(\mu \mathrm{m})$ & -1.625 & $\begin{array}{l}-5.278 \text { to } \\
2.028\end{array}$ & $<.001$ & 0.012 & 0.045 & 0.149 \\
\hline
\end{tabular}

ANTERION versus IOLMaster500

Systematic Difference

Proportional Bias

\begin{tabular}{|c|c|c|c|c|}
\hline Parameters & $\begin{array}{l}\text { Mean } \\
\text { differences }\end{array}$ & 95\% LoA & $P$ & $\begin{array}{l}\text { Scaling } \\
\text { difference }\end{array}$ \\
\hline
\end{tabular}

$\begin{array}{llllll}\mathrm{Km}(\mathrm{D}) & -0.115 & \begin{array}{l}-0.588 \text { to } \\ 0.357\end{array} & 0.002 & -0.003 & \begin{array}{l}3.41 \times 10^{-} \\ 0.902\end{array} \\ & & & & 4\end{array}$

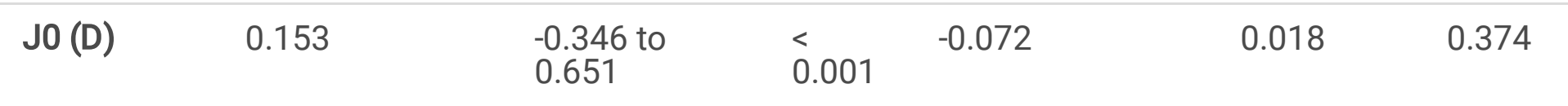

$\begin{array}{llllll}\text { J45 (D) } \quad 0.044 & \begin{array}{l}-0.398 \text { to } \\ 0.485\end{array} & 0.189 & -0.274 & 0.058 & 0.104\end{array}$

\begin{tabular}{|c|c|c|c|c|c|c|}
\hline $\mathrm{ACD}(\mathrm{mm})$ & 0.002 & $\begin{array}{l}-0.260 \text { to } \\
0.263\end{array}$ & 0.932 & 0.037 & 0.026 & 0.278 \\
\hline $\mathrm{AL}(\mathrm{mm})$ & -0.021 & $\begin{array}{l}-0.055 \text { to } \\
0.013\end{array}$ & $\begin{array}{l}< \\
0.001\end{array}$ & 0.007 & 0.260 & $\begin{array}{l}< \\
0.001\end{array}$ \\
\hline
\end{tabular}

\section{CASIA II versus IOLMaster500}

Systematic Difference

Proportional Bias

* Km, mean keratometry; J0, J0 vector components of astigmatism; J45, J45 vector components of astigmatism; ACD, anterior chamber depth; CCT, central corneal thickness; $95 \%$ LoA, 95\% limit of agreement.

$9.8 \%$ of the 10 significant results out of the 28 tests are expected to be false discoveries due to random error as determined by the false discovery rate. 


\begin{tabular}{|c|c|c|c|c|c|c|}
\hline \multirow[b]{2}{*}{ Parameters } & \multicolumn{6}{|c|}{ CASIA II vs ANTERION } \\
\hline & $\begin{array}{l}\text { Mean } \\
\text { differences }\end{array}$ & $95 \%$ LoA & $\mathrm{P}$ & $\begin{array}{l}\text { Scaling } \\
\text { difference }\end{array}$ & $\mathrm{R}^{2}$ & $\mathrm{P}$ \\
\hline $\mathrm{Km}(\mathrm{D})$ & -0.093 & $\begin{array}{l}-0.562 \text { to } \\
0.376\end{array}$ & 0.010 & -0.002 & $\begin{array}{l}9.55 \times 10^{-} \\
5\end{array}$ & 0.948 \\
\hline J0 (D) & 0.195 & $\begin{array}{l}-0.384 \text { to } \\
0.774\end{array}$ & $<.001$ & -0.116 & 0.032 & 0.232 \\
\hline J45 (D) & 0.067 & $\begin{array}{l}-0.348 \text { to } \\
0.482\end{array}$ & 0.035 & -0.201 & 0.039 & 0.185 \\
\hline ACD (mm) & 0.024 & $\begin{array}{l}-0.235 \text { to } \\
0.283\end{array}$ & 0.219 & 0.020 & 0.008 & 0.547 \\
\hline \multicolumn{7}{|c|}{$\begin{array}{l}\text { *Km, mean keratometry; J0, J0 vector components of astigmatism; J45, J45 vector components of } \\
\text { astigmatism; ACD, anterior chamber depth; CCT, central corneal thickness; } 95 \% \text { LoA, } 95 \% \text { limit of } \\
\text { agreement. }\end{array}$} \\
\hline \multicolumn{7}{|c|}{$\begin{array}{l}9.8 \% \text { of the } 10 \text { significant results out of the } 28 \text { tests are expected to be false discoveries due to } \\
\text { random error as determined by the false discovery rate. }\end{array}$} \\
\hline
\end{tabular}

\section{Discussion}

To our knowledge, this is the first study to compare the anterior segment swept-source OCT devices CASIAll and ANTERION - with the widely used PCI-based, IOLMaster500 for measurements of the corneal topography, ACD, and AL. We had also previous compared the performance of CASIAll and ANTERION for the measurement of the angle parameters ${ }^{16}$. We also acknowledged that previous publications compared Tomey CASIA SS-1000 and ANTERION for keratometry ${ }^{17}$ and other ocular biometric parameters ${ }^{18}$ but either study involve the new CASIAll that was used in our study.

Comparison between ANTERION and IOLMaster500 showed that the swept-source OCT had superior repeatability in measuring AL. There was also a significant systematic difference and proportional bias between ANTERION and IOLMaster500 for AL measurements. AL measured by the ANTERION were shorter compared to that measured by the IOLMaster 500 although a mean difference of $0.021 \mathrm{~mm}$ may not be clinically significant given that a difference in $\mathrm{AL}$ of $0.030 \mathrm{~mm}$ would only result in a spherical error of approximately $0.1 \mathrm{D}$ in eyes with average $\mathrm{AL}$ and corneal curvature ${ }^{1}$. The new IOLMaster700 (Carl Zeiss Meditec, Jena, Germany) also bases on swept-source OCT to measure AL. Some studies found no difference for AL measurements between the IOLMaster500 and $700^{19}, 20$, whereas others reported significant difference between the two devices ${ }^{21,22}$. Swept-source OCT device had demonstrated a superior ability to perform measurement compared with $\mathrm{PCl}$-based device in the case of dense cataract and posterior subcapsular cataract ${ }^{23}$. The ability to use a longer wavelength than that used by PCI $(780 \mathrm{~nm})$ can reduce light scattering from opaque media, allowing greater penetration through a severe cataract. However, our patients had an axial length in the normal range and a relatively good vision (at 
least 6/12), the performance of these devices for eyes with extreme $A L$ and dense cataract requires further study.

The ACD obtained with the ANTERION and CASIAll showed similar repeatability. There was a mean difference of $0.022 \mathrm{~mm}$ with a span of $95 \%$ LoA of $0.109 \mathrm{~mm}$. The ACD measurements obtained with the ANTERION was shorter than CASIAll. Such difference may not affect IOL power calculation because a difference of $0.100 \mathrm{~mm}$ in ACD would only lead to an approximately $0.15 \mathrm{D}$ change in refraction in eyes with an average $\mathrm{AL}$ and corneal curvature ${ }^{1}$. Either swept-source OCTs device provided more repeatable measurements of ACD compared to the IOLMaster500. There is no statistically significant systematic difference or proportional bias between the ACD of IOLMaster500 compared with either OCT. However, the span of $95 \%$ LoA were as much as $0.5 \mathrm{~mm}$ when compared with either ANTERION and CASIAll, and this can be clinically significant for IOL power calculation. Comparison between IOLMaster500 and a different swept-source OCT biometer (OA-2000, Tomey, Nagoya, Japan) also found a wide span of $95 \%$ LoA $(0.41 \mathrm{~mm})$ in ACD measurements ${ }^{24}$. Unlike the OCTs that measure the ACD from OCT image that is aligned with the optical axis of the eye, the principle of IOLMaster500 for measuring ACD is based on an optical section through the anterior chamber using a lateral slit beam illumination technique, which can be prone to operator errors ${ }^{21}$.

Although CCT measurement is not crucial for IOL power calculation, it is important for eyes planning for corneal refractive surgery. The CCT obtained with the two swept-source OCTs demonstrated similar repeatability but a significant systematic difference of $1.6 \mu \mathrm{m}$ with a span of $95 \%$ LoA of $7.3 \mu \mathrm{m}$. A much wider span of $95 \%$ LoA of $35 \mu \mathrm{m}$ has been found between two other swept-source OCT based biometers, IOLMaster 700 and ARGOS (Movu, Komaki, Japan) ${ }^{25}$. The reason for the differences could be the inconsistent measurement location and different measurement strategies between the different sweptsource OCTs.

The $\mathrm{Km}$ measurements demonstrated comparable repeatability for all three devices. However, the Km measured by IOLMaster500 showed a systematic difference when compared with either ANTERION or CASIAll, implying between-device disagreement. The $\mathrm{Km}$ measured by IOLMaster500 was significantly steeper compared with ANTERION (0.115 D) and CASIAll (0.093 D). It has been suggested that a Km difference of $0.20 \mathrm{D}$ between the devices is sufficient to give different optimized constants for IOL power calculation ${ }^{26}$. Intraocular lens constant optimization based on the difference in magnitude in $\mathrm{Km}$ values has been proposed. Karunaratne et al demonstrated that the keratometry readings of the IOLMaster and Pentacam (Oculus, Wetzlar, Germany) were not equivalent, but their keratometry readings were similarly effective in IOL power calculations after constant optimization ${ }^{27}$. Similarly, although our findings showed that the $\mathrm{Km}$ measured by IOLMaster500 significantly differs from either swept-source OCTs, such disagreement could be refined by readjustment of intraocular lens constant in clinical practice.

As for the $\mathrm{J} 0$ and $\mathrm{J} 45$ astigmatic vector measurements, the $\mathrm{J} 0$ measured by ANTERION demonstrated a significantly lower value of RC compared with CASIAll, and a similar RC for J45. Although the RC of J0 and J45 obtained with IOLMaster500 did not show a significant difference compared with either OCT 
(apart from a significantly higher RC for J0 when compared with the ANTERION), there is a trend of higher $\mathrm{RC}$ values for the vector components obtained with the IOLMaster500. The between-instrument agreement of IOLMaster 500 and either OCT for the astigmatic vector measurements was poor. There was a systematic difference for the J0 obtained with the IOLMaster500 compared with either the ANTERION or CASIAll. A systematic difference was also found for the $\mathrm{J} 45$ between the IOLMaster 500 and CASIAll. These observations are clinically important, given that the accuracy of astigmatism measurements is crucial for the implantation toric IOL. Our data suggested that ANTERION demonstrated superior repeatability for the measurement of $\mathrm{J} 0$, which represents with-the-rule and against-the-rule astigmatism, and its agreement with the IOLMaster500 was poor. In practice, it is important to perform corneal topography when before implanting a toric IOL in cataract surgery since irregular corneal astigmatism,

which is not correctable by toric IOL, has been shown to increase with increasing age $\mathrm{e}^{28}$. It is likely that the difference between PCI-based and swept-source OCT based devices exists because of the different methods used to analyze the mires of spots reflected from the cornea. The IOLMaster500 measures keratometry from the anterior cornea in the $2.5 \mathrm{~mm}$ zone using only 6 spots of light projected on the cornea ${ }^{19}$, which is different from swept-source OCTs that utilizes multiple evenly spaced radial B-scans for the measurement ${ }^{19}$. Therefore, the astigmatic axis measured by the swept-source OCT and the PClbased IOLMaster500 should not be considered as interchangeable. It remains to be elucidated how the differences of the measurements would affect the predicted residual astigmatism after toric IOL implantation.

This study has several limitations. We did not grade the severity of cataract in our patients. Previous study showed that swept-source OCT-based device was more effective in obtaining biometric measurements in eyes with posterior subcapsular and dense nuclear cataract ${ }^{21}$. Our study did not recruit patients with visual acuity worse than Snellen 6/12. Our study did not investigate the refractive outcome after cataract surgery; we, therefore, could not determine the refractive prediction errors because of the measurement differences in IOL power calculation. Further study is required to verify how the findings in the current study that may affect the cataract surgical outcome in clinical practice.

In conclusion, both ANTERION and CASIAll demonstrated excellent repeatability in biometry and corneal measurements. Favourable agreement was demonstrated in keratometry and its astigmatic axis measurements between the two swept-source OCTs. On the other hand, IOLMaster500 demonstrated a lower repeatability for $A C D$ and $A L$ measurements. There was significant disagreement in keratometry and AL measurements between swept-source OCT and PCI-based devices.

\section{Declarations}

Conflict of interest: CL has received research support in the form of instrument and speaker honorarium from Tomey (Japan) and Heidelberg Engineering (Germany)

\section{Acknowledgments/Disclosure}




\section{Funding/Support}

None

\section{Financial Disclosures}

No financial disclosures

\section{References}

1. Norrby S. Sources of error in intraocular lens power calculation. J Cataract Refract Surg 2008;34:368376.

2. Chen YA, Hirnschall N, Findl O. Evaluation of 2 new optical biometry devices and comparison with the current gold standard biometer. J Cataract Refract Surg 2011;37:513-517.

3. Kaswin G, Rousseau A, Mgarrech M, Barreau E, Labetoulle M. Biometry and intraocular lens power calculation results with a new optical biometry device: comparison with the gold standard. $J$ Cataract Refract Surg 2014;40:593-600.

4. Olsen T. Improved accuracy of intraocular lens power calculation with the Zeiss IOLMaster. Acta Ophthalmol Scand 2007;85:84-87.

5. Kunavisarut P, Poopattanakul P, Intarated C, Pathanapitoon K. Accuracy and reliability of IOL master and A-scan immersion biometry in silicone oil-filled eyes. Eye (Lond) 2012;26:1344-1348.

6. Fan R, Chan TC, Prakash G, Jhanji V. Applications of corneal topography and tomography: a review. Clin Exp Ophthalmol 2018;46:133-146.

7. Radhakrishnan S, Rollins AM, Roth JE, et al. Real-time optical coherence tomography of the anterior segment at $1310 \mathrm{~nm}$. Arch Ophthalmol 2001;119:1179-1185.

8. Liu S, Yu M, Ye C, Lam DS, Leung CK. Anterior chamber angle imaging with swept-source optical coherence tomography: an investigation on variability of angle measurement. Invest Ophthalmol Vis Sci 2011; 52:8598-8603.

9. Lai I, Mak H, Lai G, Yu M, Lam DS, Leung CK. Anterior chamber angle imaging with swept-source optical coherence tomography: measuring peripheral anterior synechia in glaucoma. Ophthalmology 2013;120:1144-1149.

10. Tomey. Fourier Domain OCT CASIA2: 3D swept source OCT. Available at: https://tomey.de/images/product_flyer/CASIA2_br_w.pdf. Assess March 15, 2020. 2016.

11. Teussink MM, Donner S, Otto T, Williams K, Tafreshi A. State-of-art commercial Spectral Domain and Swept-Source OCT technologies and their clinical applications in ophthalmology. Heidelberg Enginnering Academy 2019;1-19. Available at: https://businesslounge.heidelbergengineering.com/gf/en/products/anterion/anterion/publications/\#publications. Access July 12, 2019. 
12. Asam JS, Polzer M, Tafreshi A, Hirnschall N, Findl O. Anterior Segment OCT: High Resolution Imaging in Microscopy and Ophthalmology: Springer, Cham; 2019.

13. Thibos LN, Wheeler W, Horner D. Power vectors: an application of Fourier analysis to the description and statistical analysis of refractive error. Optom Vis Sci 1997;74:367-375.

14. Bland JM, Altman DG. Measurement error. BMJ 1996;312:1654.

15. Benjamini Y, Hochberg Y. Controlling the False Discovery Rate: A Practical and Powerful Approach to Multiple Testing Journal of the Royal Statistical Society Series B (Methodological) 1995;57:289-300.

16. Chan PP, Lai G, Chiu V, Chong A, Yu M, Leung CK. Anterior chamber angle imaging with swept-source optical coherence tomography: comparison between CASIAll and ANTERION. Sci Rep 2020;10:18771.

17. Gjerdrum B, Gundersen KG, Lundmark PO, Aakre BM. Repeatability of OCT-Based versus Scheimpflugand Reflection-Based Keratometry in Patients with Hyperosmolar and Normal Tear Film. Clin Ophthalmol 2020;14:3991-4003.

18. Pardeshi AA, Song AE, Lazkani N, Xie X, Huang A, Xu BY. Intradevice Repeatability and Interdevice Agreement of Ocular Biometric Measurements: A Comparison of Two Swept-Source Anterior Segment OCT Devices. Trans/ Vis Sci Technol 2020;9:14.

19. Srivannaboon S, Chirapapaisan C, Chonpimai P, Loket S. Clinical comparison of a new swept-source optical coherence tomography-based optical biometer and a time-domain optical coherence tomography-based optical biometer. J Cataract Refract Surg 2015;41:2224-2232.

20. Kunert KS, Peter M, Blum M, et al. Repeatability and agreement in optical biometry of a new sweptsource optical coherence tomography-based biometer versus partial coherence interferometry and optical low-coherence reflectometry. J Cataract Refract Surg 2016;42:76-83.

21. Akman A, Asena L, Gungor SG. Evaluation and comparison of the new swept source OCT-based IOLMaster 700 with the IOLMaster500. Br J Ophthalmol 2016;100:1201-1205.

22. Yang JY, Kim HK, Kim SS. Axial length measurements: Comparison of a new swept-source optical coherence tomography-based biometer and partial coherence interferometry in myopia. J Cataract Refract Surg 2017;43:328-332.

23. McAlinden C, Wang Q, Gao R, et al. Axial Length Measurement Failure Rates With Biometers Using Swept-Source Optical Coherence Tomography Compared to Partial-Coherence Interferometry and Optical Low-Coherence Interferometry. Am J Ophthalmol 2017;173:64-69.

24. Huang J, Savini G, Hoffer KJ, et al. Repeatability and interobserver reproducibility of a new optical biometer based on swept-source optical coherence tomography and comparison with IOLMaster. $\mathrm{Br} J$ Ophthalmol 2017;101:493-498.

25. Sabatino F, Matarazzo F, Findl O, Maurino V. Comparative analysis of 2 swept-source optical coherence tomography biometers. J Cataract Refract Surg 2019;45:1124-1129.

26. Ozyol P, Ozyol E. Agreement Between Swept-Source Optical Biometry and Scheimpflug-based Topography Measurements of Anterior Segment Parameters. Am J Ophthalmol 2016;169:73-78.

27. Karunaratne N. Comparison of the Pentacam equivalent keratometry reading and IOL Master keratometry measurement in intraocular lens power calculations. Clin Exp Ophthalmol2013;41:825- 
834.

28. Hayashi K, Kawahara S, Manabe S, Hirata A. Changes in Irregular Corneal Astigmatism With Age in Eyes With and Without Cataract Surgery. Invest Ophthalmol Vis Sci 2015;56:7988-7998.

\section{Figures}

(A)

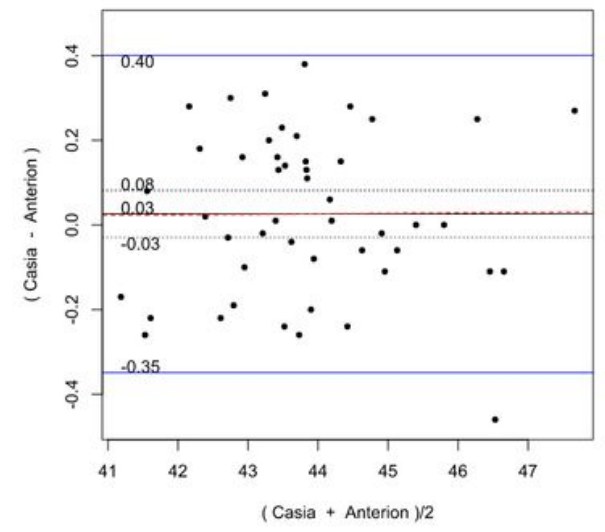

(B)

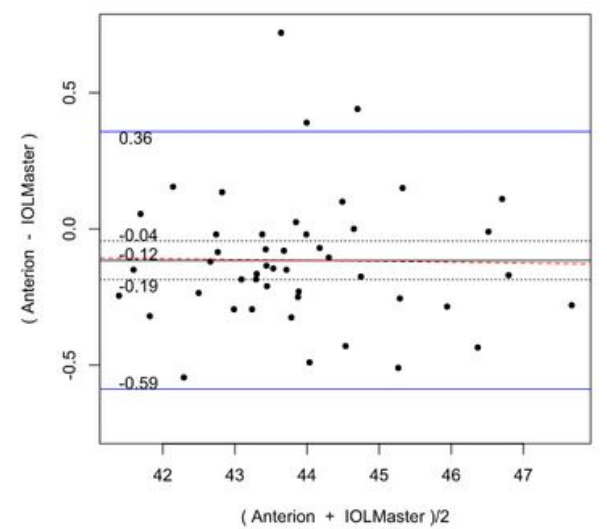

(C)

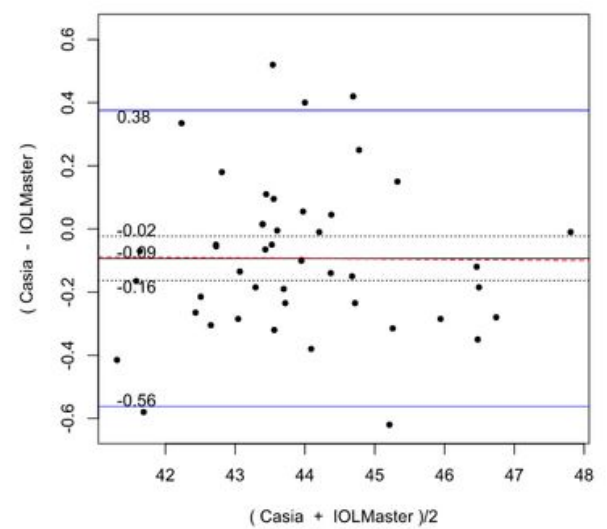

Figure 1

Bland-Altman plots showing the pairwise agreement between ANTERION vs CASIAII (A), ANTERION vs IOLMaster500 (B), and CASIAIl vs IOLMaster500 (C) for mean keratometry (Km)

(A)

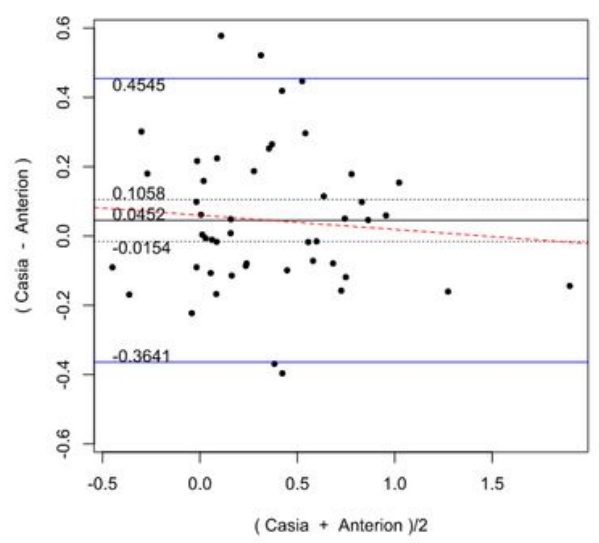

(B)

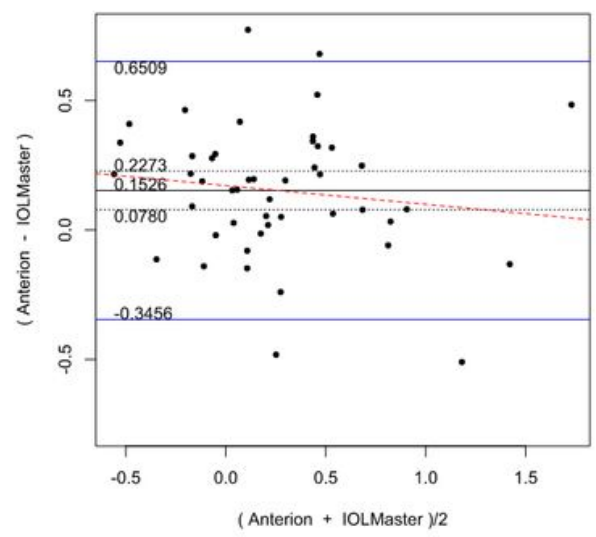

(C)

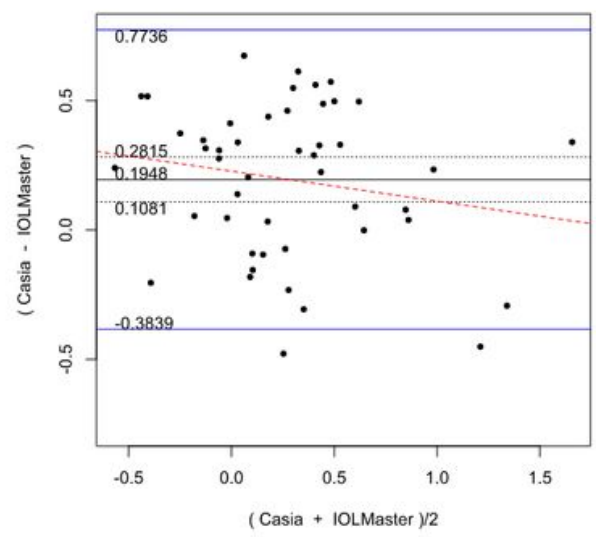

Figure 2

Bland-Altman plots showing the pairwise agreement between ANTERION vs CASIAII (A), ANTERION vs IOLMaster500 (B), and CASIAll vs IOLMaster500 (C) for J0 vector component of astigmatism 
(A)

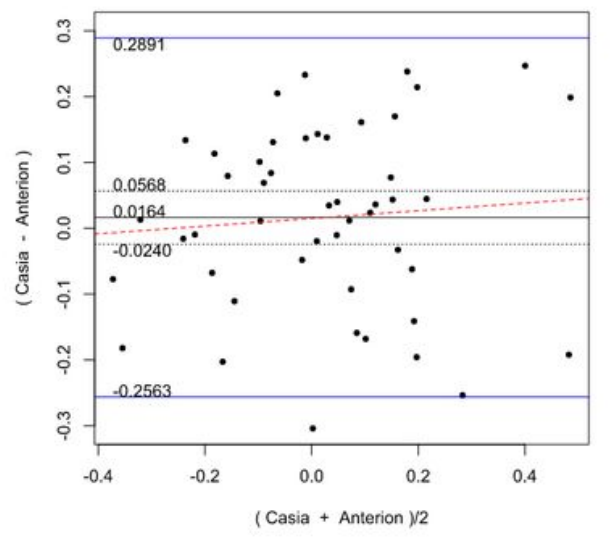

(B)

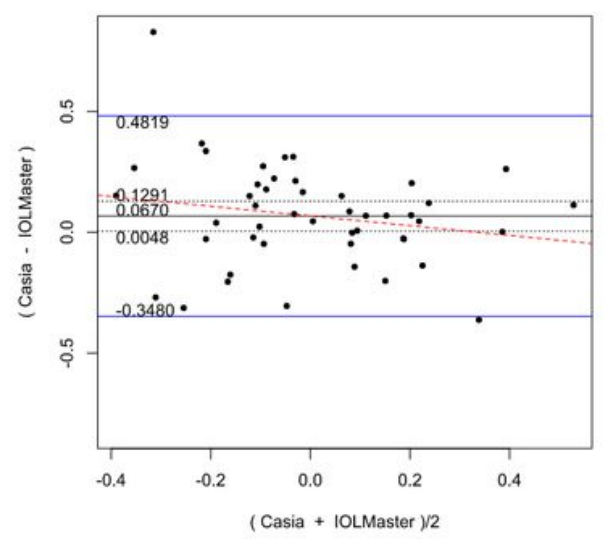

(C)

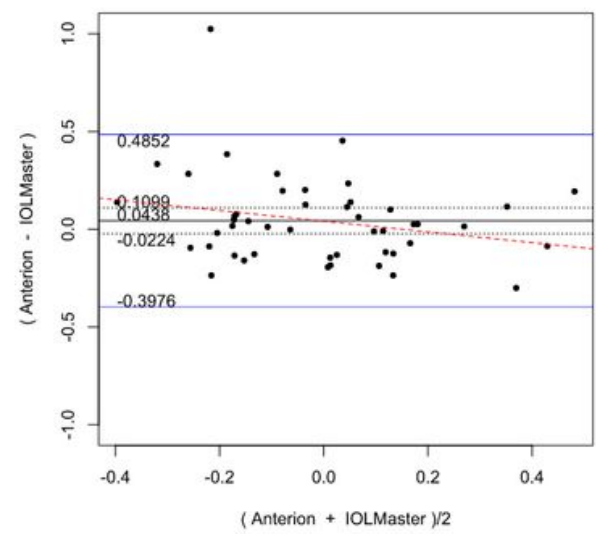

\section{Figure 3}

Bland-Altman plots showing the pairwise agreement between ANTERION vs CASIAll (A), ANTERION vs IOLMaster500 (B), and CASIAll vs IOLMaster500 (C) for J45 vector component of astigmatism

(A)

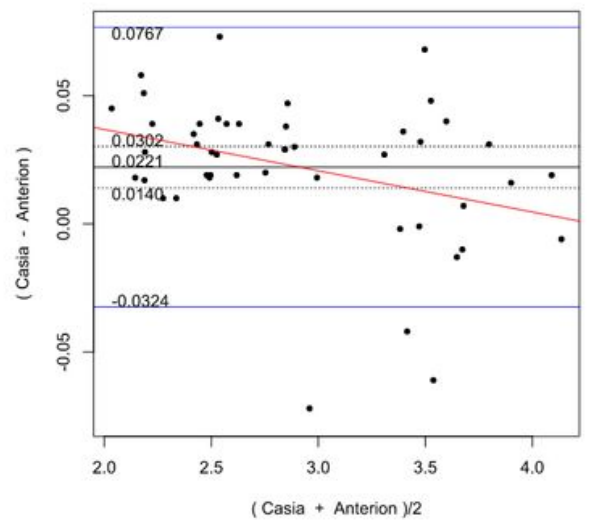

(B)

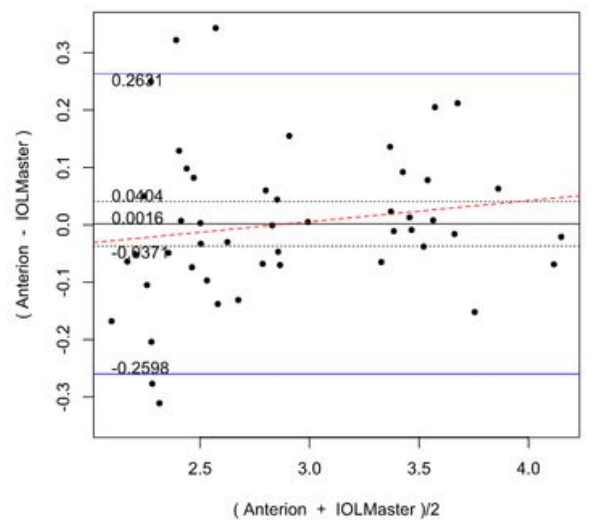

(C)

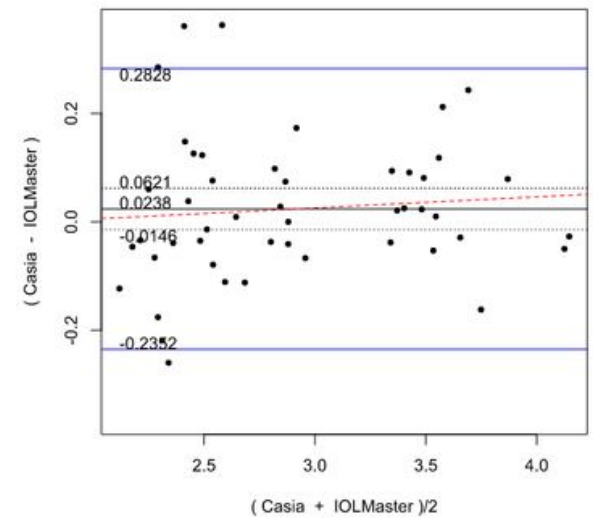

Figure 4

Bland-Altman plots showing the pairwise agreement between ANTERION vs CASIAll (A), ANTERION vs IOLMaster500 (B), and CASIAll vs IOLMaster500 (C) for anterior chamber depth (ACD) 


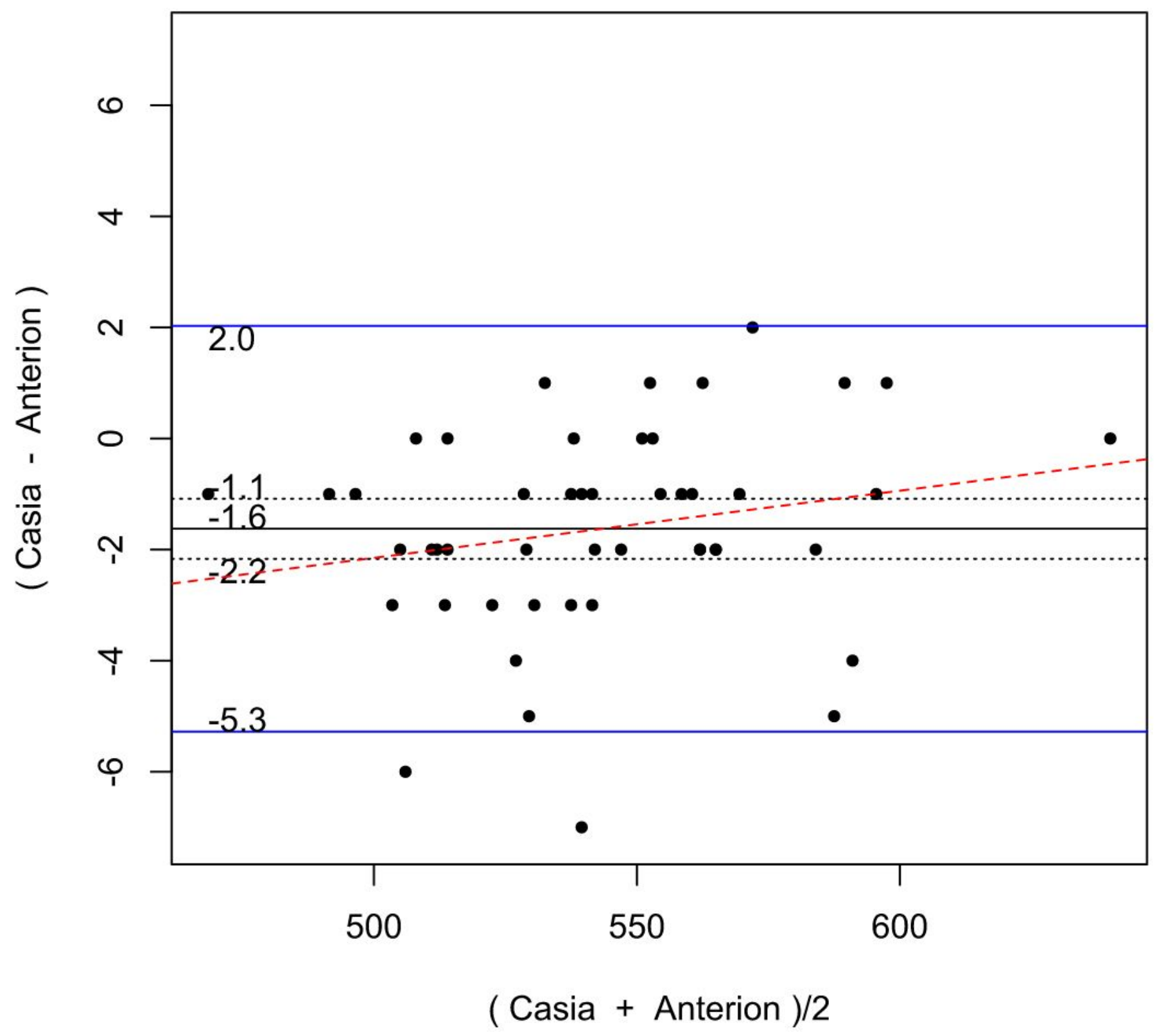

Figure 5

Bland-Altman plots showing the pairwise agreement between ANTERION vs CASIAll for central corneal thickness (CCT) 


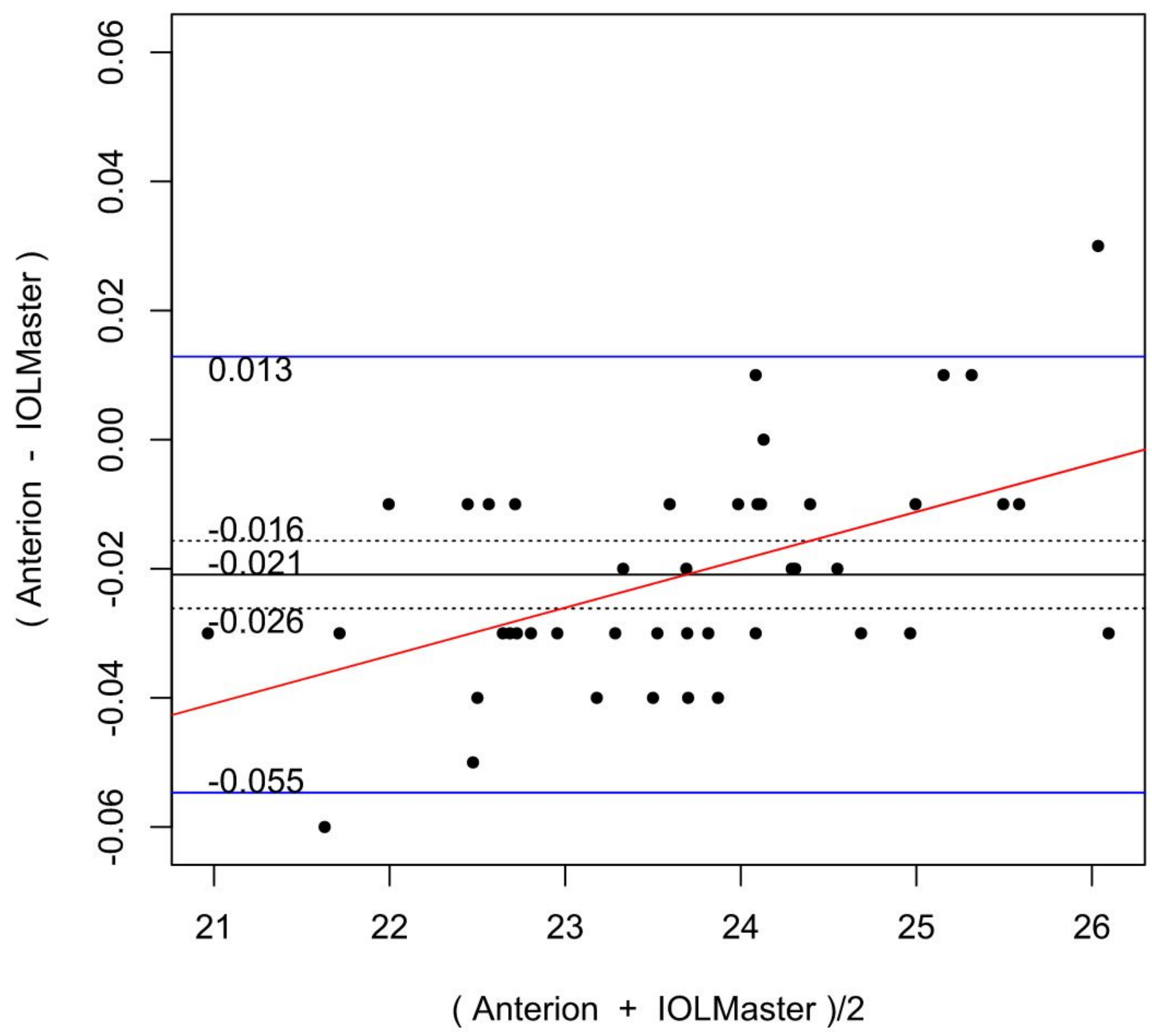

Figure 6

Bland-Altman plots showing the pairwise agreement between ANTERION vs IOLMaster500 for axial length $(\mathrm{AL})$ 